\title{
PAPER
}

\section{On existence and uniqueness of a carrying simplex in Kolmogorov differential systems}

To cite this article: Zhanyuan Hou 2020 Nonlinearity 337067

View the article online for updates and enhancements. 


\title{
On existence and uniqueness of a carrying simplex in Kolmogorov differential systems
}

\author{
Zhanyuan Hou \\ School of Computing and Digital Media, London Metropolitan University, 166-220 \\ Holloway Road, London N7 8DB, United Kingdom \\ E-mail: z.hou@londonmet.ac.uk
}

Received 8 January 2020, revised 21 July 2020

Accepted for publication 18 August 2020

Published 9 November 2020

\begin{abstract}
This paper deals with global asymptotic behaviour of the dynamics for $N$-dimensional competitive Kolmogorov differential systems of equations $\frac{\mathrm{d} x_{i}}{\mathrm{~d} t}=x_{i} f_{i}(x), \quad 1 \leqslant i \leqslant N, x \in \mathbb{R}_{+}^{N}$. A theory based on monotone dynamical systems was well established by Hirsch (1988 Nonlinearity 1 51-71). One of his theorems is outstanding and states the existence of a co-dimension 1 compact invariant submanifold $\Sigma$ that attracts all the nontrivial orbits under certain assumptions and, in practice, under the condition that the system is totally competitive (all $N^{2}$ entries of the Jacobian matrix $D f$ are negative). The submanifold $\Sigma$ has been called carrying simplex since then and the theorem has been well accepted with many hundreds of citations. In this paper, we point out that the requirement of total competition is too restrictive and too strong; we prove the existence and uniqueness of a carry simplex under the assumption of strong internal competition only (i.e. $N$ diagonal entries of $D f$ are negative), a much weaker condition than total competition. Thus, we improve the theorem significantly by dramatic cost reduction from requiring $N^{2}$ to $N$ negative entries of $D f$. As an example of applications of the main result, the existence and global attraction (repulsion) of a heteroclinic limit cycle for three-dimensional systems is discussed and two concrete examples are given to demonstrate the existence of such heteroclinic cycles.
\end{abstract}

Keywords: competitive Kolmogorov systems, carrying simplex, existence and uniqueness, heteroclinic limit cycles, global attraction, global repulsion Mathematics Subject Classification numbers: 37C70, 34D45, 34C45, 34C12, 34D23, 37C75, 37B25, 92D25. 


\section{Introduction}

In this paper, we are concerned with the global dynamical behaviour of the flow $\varphi_{t}$ generated by the $N$-dimensional Kolmogorov differential system of equations

$$
\dot{x}_{i}=x_{i} f_{i}(x)=F_{i}(x), \quad x \in C, i \in I_{N}=\{1,2, \ldots, N\},
$$

where $C=\mathbb{R}_{+}^{N}=\left\{x \in \mathbb{R}^{N}: \forall i \in I_{N}, x_{i} \geqslant 0\right\}$ and $f \in C^{1}\left(C, \mathbb{R}^{N}\right)$. System (1) is a typical mathematical model for the population dynamics of a community of $N$ species, where each $x_{i}(t)$ represents the population size or density at time $t$ and the function $f_{i}(x)$ denotes the per capita growth rate of the $i$ th species. For this reason, system (1) is called cooperative if $\frac{\partial f_{i}(x)}{\partial x_{j}} \geqslant 0$ for all $i, j \in I_{N}$ with $i \neq j$ because increase of the $j$ th population boosts the per capita growth rate of the $i$ th species. Similarly, system (1) is called competitive if $\frac{\partial f_{i}(x)}{\partial x_{j}} \leqslant 0$ for all $i, j \in I_{N}$ with $i \neq j$ as increase of the $j$ th population reduces the per capita growth rate of the $i$ th species. A competitive system is called totally competitive if $\frac{\partial f_{i}(x)}{\partial x_{j}}<0$ for all $i, j \in I_{N}$. We say that a competitive system has strong internal competition if $\frac{\partial f_{i}(x)}{\partial x_{i}}<0$ for all $i \in I_{N}$. System (1) is called dissipative if there is a compact invariant set $A \subset C$ that uniformly attracts each compact set of initial values. A square matrix $A$ is said to be irreducible if there is no permutation matrix $P$ such that

$$
P^{\mathrm{T}} A P=\left(\begin{array}{cc}
A_{11} & A_{12} \\
0 & A_{22}
\end{array}\right)
$$

where $A_{11}$ and $A_{22}$ are square matrices.

System (1) and its various particular instances, such as the classic Lotka-Volterra systems, have attracted huge interests from researchers spanning in the last half century. Consequently, numerous research results for such systems can be found in literature. For example, Hirsch [7-10] investigated the dynamical behaviour of cooperative and competitive systems (1) in line with the development of the theory on monotone dynamical systems. The following is one of his theorems given in [9].

Theorem 1.1. Assume that (1) satisfies the following conditions:

(a) The system is competitive.

(b) The system is dissipative.

(c) The Jacobian matrix $D f(x)$ is irreducible in $C$.

(d) The origin 0 is a repellor.

(e) At each nonzero equilibrium, every entry of $D f$ is negative.

Then (1) has a compact invariant submanifold $\Sigma \subset C$ homeomorphic to $\Delta^{N-1}=\{x \in$ $\left.C: x_{1}+\cdots+x_{N}=1\right\}$ by radial projection such that every nontrivial trajectory in $C$ is asymptotic to one in $\Sigma$.

This theorem is outstanding, phenomenal and has a big impact to later on researches on competitive systems due to the important and interesting features of the set $\Sigma$ : compact, invariant, unordered ( $p \leqslant q$ implies $p=q$ for $p, q \in \Sigma$ ), homeomorphic to $\Delta^{N-1}$ by radial projection, and a global attractor of (1) in $C \backslash\{0\}$. This theorem proves that the dynamical behaviour of the system in $C$ is essentially described by that of the system on this co-dimension 1 surface $\Sigma$. Zeeman [42] called $\Sigma$ the carrying simplex and it has been known as carrying simplex since then.

There is a wide range of applications of this theorem with many hundreds of citations in literature. Although a thorough investigation on these citations is not our intention here, 
research publications citing this theorem roughly fall into the following four categories: 1 . Some researchers further investigated the geometry of $\Sigma$. For example, Zeeman and Zeeman [40], Tineo [35], Baigent [1, 2] investigated convexity of $\Sigma$; Mierczyński [30-33] studied the smoothness of $\Sigma$. 2. Some researchers based their work on the existence of a carrying simplex. For example, Zeeman and Zeeman [41], Hou and Baigent [5, 18, 20], and Hou [14] investigated global stability and repulsion of a fixed point; Hou and Baigent [19] studied the existence and global stability of heteroclinic limit cycles; Zeeman [42], Jiang and Niu [22-24] and Jiang et al [28] investigated the dynamical behaviour on carrying symplex. 3. Some research projects were inspired by Hirsch's theorem although they could not use theorem 1.1 because some of its conditions were not met. For example, Hou [11-13, 15-17] investigated permanence and stability without assuming the existence of a carrying simplex; Liang and Jiang [29] studied the dynamical behaviour of type-K competitive systems; Mierczyński and Schreiber [34] established permanence conditions; Tu and Jiang [36] found coexistence conditions for systems with limited competition; Zeeman [43] picked up a condition for extinction for some species; Yu et al [39] gave a criterion for global stability of three-dimensional system. 4 . The concept of carrying simplex has been extended to discrete competitive dynamical systems. Research in this area has been flourishing in the last two decades. Typical examples are $[3,4,6,10,21$, 25-27, 37, 38].

We note that users of theorem 1.1 seem scared of the situation when $\frac{\partial f_{i}(x)}{\partial x_{j}}=0$ for some $i \neq j$ and some $x \in C$ due to the requirements of (c) and (e). Since total competition implies the fulfilment of conditions (c) and (e), in practice, almost all users of theorem 1.1 assume total competition for convenience. We are reluctant to face the reality that the requirement of total competition is very restrictive and costly. But actually, conditions (c) and (e) are not necessary for the existence and uniqueness of a carrying simplex.

The aim of this paper is to prove the existence and uniqueness of a carrying simplex under a much weaker condition than (c) and (e): we only require that system (1) has strong internal competition on a globally attracting compact positive invariant set rather than total competition in $C$. We shall present our main theorem in section 2 and prove it in section 3 . In section 4 we present a result on existence of globally attracting or repelling heteroclinic limit cycles and two concrete examples for three-dimensional systems as an application of the main theorem. We then conclude the paper in section 5 .

\section{Notation and statement of the main result}

For $C=\mathbb{R}_{+}^{N}$ we let $\dot{C}=\left\{x \in C: \forall i \in I_{N}, x_{i}>0\right\}$ and $\partial C=C \backslash \dot{C}$. Then $\dot{C}$ is the interior of $C$ and $\partial C$ is the boundary of $C$. The part of $\partial C$ restricted to the $i$ th coordinate plane and the part restricted to the positive half $x_{i}$-axis are denoted by $\pi_{i}$ and $X_{i}$ respectively, i.e.

$$
\begin{aligned}
& \pi_{i}=\left\{x \in C: x_{i}=0\right\}, i \in I_{N}, \\
& X_{i}=\left\{x \in C: x_{i}>0, \forall j \in I_{N} \backslash\{i\}, x_{j}=0\right\}, i \in I_{N} .
\end{aligned}
$$

Denote the $i$ th standard unit vector by $e_{i}$, i.e. the $i$ th component of $e_{i}$ is 1 and others are 0 . For any nonempty subset $I \subset I_{N}$, define

$$
\begin{aligned}
& C_{I}=\left\{x \in C: \forall j \in I_{N} \backslash I, x_{j}=0\right\}, \\
& \dot{C}_{I}=\left\{x \in C_{I}: \forall i \in I, x_{i}>0\right\} .
\end{aligned}
$$

For any $x, y \in C_{I}$, by writing $x \leqslant_{I} y$ we mean $x_{i} \leqslant y_{i}$ for all $i \in I$; we write $x<_{I} y$ if $x \leqslant \leqslant_{I} y$ but $x \neq y$; and we write $x \ll_{I} y$ if $y-x \in \dot{C}_{I}$. We may also use $y \geqslant_{I} x, y>_{I} x$ and $y \gg_{I} x$ for 
$x \leqslant_{I} y, x<_{I} y$ and $x \ll_{I} y$ respectively. If $I=I_{N}$, we simply drop the subscript ' $I$ ' from these inequalities. For any $a, b \in C$ with $a \leqslant b$, we let $[a, b]=\{x \in C: a \leqslant x \leqslant b\}$. Then $[a, b]$ is a $k$-dimensional cell if $b-a$ has exactly $k$ positive components. If $a, b \in C_{I}$ for some $I \subset I_{N}$ with $|I|=k \leqslant N$ such that $a \ll_{I} b$, we define $(a, b)=\left\{x \in C_{I}: a \ll_{I} x \ll_{I} b\right\}$. Then $(a, b)$ is an open cell in $\mathbb{R}^{k}$ with closure $[a, b]$. For each $x \in C$, the positive and negative limit sets under the flow $\varphi_{t}$, with usual definition, are denoted by $\omega(x)$ and $\alpha(x)$ respectively. For convenience, we define the concept of a carrying simplex as follows.

Definition 2.1. A nonempty compact set $\Sigma \subset C \backslash\{0\}$ is called a carrying simplex of (1) if $\Sigma$ meets the following requirements.

(a) $\Sigma$ is an invariant submanifold homeomorphic to $\Delta^{N-1}$ by radial projection.

(b) For each $x \in C \backslash\{0\}$, there is a $y \in \Sigma$ such that $\lim _{t \rightarrow+\infty}\left(\varphi_{t}(x)-\varphi_{t}(y)\right)=0$.

Note that the 'unordered' property of $\Sigma$ is not mentioned in the above definition. We shall see in remark 2.1(e) below that the unordered property of $\Sigma$ here is slightly different from that in Hirsch [9], Zeeman [42] and the literature.

Theorem 2.2. Assume that system (1) satisfies the following conditions:

(a) $f(0) \gg 0$.

(b) There exists a vector $r \gg 0$ such that

$$
\forall i \in I_{N}, \forall x \in C \quad \text { with } x_{i} \geqslant r_{i}, f_{i}(x)<0 \text {. }
$$

(c) $f \in C^{1}\left([0, r], \mathbb{R}^{N}\right)$ and

$$
\forall x \in[0, r], \forall i, j \in I_{N}, \quad \frac{\partial f_{i}(x)}{\partial x_{i}}<0 \quad \text { and } \quad \frac{\partial f_{i}(x)}{\partial x_{j}} \leqslant 0 .
$$

Then (1) has a unique carrying simplex $\Sigma$. Moreover, for each $p \in \Sigma$ and every $q \in[0, r] \backslash\{0\}$ with $q<p$, we have $\alpha(q) \subset \pi_{i}$ provided $q_{i}<p_{i}$.

\section{Remark 2.1.}

(a) Condition (a) of theorem 2.2 implies that the origin is a repellor, which is the same as condition (d) of theorem 1.1 .

(b) Condition (b) can be interpreted as ' $\infty$ ' is a source so the system is dissipative with a global attractor $A \subset[0, r]$. This is essentially the same as condition (b) of theorem 1.1.

(c) Condition (c) requires the system to be competitive with strong internal competition on $[0, r]$, which is much weaker than (a), (c) and (e) together in theorem 1.1.

(d) For each $i \in I_{N}$, conditions (a)-(c) imply that the one-dimensional subsystem $\dot{x}_{i}=$ $x_{i} f_{i}\left(x_{i} e_{i}\right)$ has a unique positive equilibrium $Q_{i}=q_{i} e_{i} \in X_{i}$ that is globally attracting on $X_{i}$. From the conclusion of theorem 2.2 we see that $\Sigma \cap X_{i}=\left\{Q_{i}\right\}$.

(e) From the conclusion we see that for each $p \in \Sigma$, there is a nonempty $I \subset I_{N}$ such that $p \in \dot{C}_{I}$. Then, for each $q \in[0, r]$ with $q \ll_{I} p$, we have $\alpha(q) \subset \pi_{i}$ for all $i \in I$. As $q \ll_{I} p$ and $p \in \dot{C}_{I}$ imply that $q \in C_{I}$, we have $\alpha(q) \subset \cap_{i=1}^{N} \pi_{i}=\{0\}$, so $\alpha(q)=\{0\} \not \subset \Sigma$. This shows that $\Sigma$ is unordered in a strict sense: for any nonempty $I \subset I_{N}$, any $p \in \dot{C}_{I}$ and any $q \ll_{I} p$, we cannot have both $p \in \Sigma$ and $q \in \Sigma$. However, due to the possibility of $\frac{\partial f_{i}(x)}{\partial x_{j}}=0$ for some $i \neq j$ and some $x, \Sigma$ does allow ordered points on it, i.e. $p, q \in \Sigma$ with $p<q$. This is demonstrated by the trivial example below. 
Example Consider the system

$$
\dot{x}_{i}=x_{i} g_{i}\left(x_{i}\right), \quad i \in I_{N},
$$

where each $g_{i}: \mathbb{R}_{+} \rightarrow \mathbb{R}$ is continuous, $g_{i}(u)<0$ for $u>q_{i}>0, g_{i}\left(q_{i}\right)=0, g_{i} \in C^{1}\left(\left[0, q_{i}\right], \mathbb{R}\right)$ and $g_{i}^{\prime}(u)<0$ for $u \in\left[0, q_{i}\right]$. Then (2) satisfies all the conditions of theorem 2.2, so it has a unique carrying simplex $\Sigma$. Note that (2) is a trivial case of (1) when there is no interaction between distinct component equations of the system. Since $q_{i}$ is the globally attracting equilibrium of the $i$ th component equation on the positive $x_{i}$-axis, $\Sigma$ is the upper boundary surface of the cell $[0, q]$, i.e.

$$
\Sigma=\left\{x \in[0, q]: x_{i}=q_{i} \text { for some } i \in I_{N}\right\} .
$$

Clearly, $q \in \Sigma$ and for each $p \in \Sigma \backslash\{q\}$, we have $p<q$. Thus, ordered points are permitted on $\Sigma$.

Corollary 2.3. Under the conditions of theorem 2.2, the following conclusions hold.

(a) For any periodic orbit $\gamma \subset \Sigma$, the points on $\gamma$ are unordered, i.e. if $p, q \in \gamma$ with $p \leqslant q$ then $p=q$.

(b) For any $x \in \Sigma$, if there are two points $p, q \in \gamma(x)$ satisfying $p<q$ then $\alpha(x)$ consists of either a single equilibrium or a periodic orbit.

\section{Proof.}

(a) Suppose there are two points $p, q \in \gamma$ satisfying $p<q$. Then there is at least one $i \in I_{N}$ such that $p_{i}<q_{i}$. From theorem 2.2 we have $\alpha(p) \subset \pi_{i}$, so $q \notin \alpha(p)$, a contradiction to $q \in \gamma=\alpha(p)$ due to the periodicity of $\gamma$. Therefore, $\gamma$ is unordered.

(b) By $x \in \Sigma$ we have $\gamma(x) \subset \Sigma$ and $\alpha(x) \subset \Sigma$. Then, by $p<q$ and the monotone property of the backward orbit for competitive systems, we have $\varphi_{t}(p)<\varphi_{t}(q)$ for all $t \leqslant 0$. Then, for each $i \in I_{N}$, if there is a $t \leqslant 0$ such that $\left(\varphi_{t}(p)\right)_{i}<\left(\varphi_{t}(q)\right)_{i}$, by theorem 2.2 we have $\alpha(x)=\alpha(p) \subset \pi_{i}$; otherwise, we have $\left(\varphi_{t}(p)\right)_{i}=\left(\varphi_{t}(q)\right)_{i}$ for all $t \leqslant 0$. Thus, there is a proper subset $I \subset I_{N}$ such that $\alpha(x) \subset \pi_{i}$ for each $i \in I$ and $\left(\varphi_{t}(p)\right)_{j}=\left(\varphi_{t}(q)\right)_{j}$ for all $t \leqslant 0$ and $j \in I_{N} \backslash I$. As $p$ and $q$ are two distinct points on $\gamma(x)$, there is a $T>0$ such that either $\varphi_{T}(p)=q$ or $\varphi_{T}(q)=p$. Hence, since the component $\left(\varphi_{t}(p)\right)_{j}$ is a $T$-periodic function for $t \leqslant 0$ for each $j \in I_{N} \backslash I$, we obtain

$$
\alpha(x)=\left\{y(t): t \in[-T, 0], y_{i}(t)=0, i \in I ; y_{j}(t)=\left(\varphi_{t}(p)\right)_{j}, j \in I_{N} \backslash I\right\}
$$

Therefore, $\alpha(x)$ consists of either a single equilibrium or a periodic orbit.

For any unit vector $u \gg 0$, let $E=\left\{x \in \mathbb{R}^{N}: u \cdot x=0\right\}$. Then $E$ is the hyperplane orthogonal to $u$. Let $P: \mathbb{R}^{N} \rightarrow E$ be the orthogonal projection. Then

$$
\forall x \in \mathbb{R}^{N}, \quad P(x)=x-(u \cdot x) u .
$$

Let $g=\left.P\right|_{\Sigma}$, the restriction of $P$ to the carrying simplex $\Sigma$.

Corollary 2.4. Under the conditions of theorem 2.2, the flow $\varphi_{t}$ on $\Sigma$ is conjugate, via the Lipschitz homeomorphism g, to the flow $\theta_{t}$ of a Lipschitz vector field on a closed set of $\mathrm{E}$ for any given unit vector $u \gg 0$, that is,

$$
\theta_{t}=g \circ \varphi_{t} \circ g^{-1} ; \quad \forall x \in g(\Sigma) \subset E, \frac{\mathrm{d}}{\mathrm{d} t}\left(\theta_{t}(x)\right)=G\left(\theta_{t}(x)\right),
$$


where $G(y)=P \circ F \circ g^{-1}(y)$

Note that corollary 2.4 is consistent with theorem $2.1^{\prime}$ given in [9]. We leave the proofs of theorem 2.2 and corollary 2.4 to the next section.

\section{Proofs of theorem 2.2 and corollary 2.4}

In order to prove theorem 2.2, we first prove a few lemmas as preparation. The first lemma identifies the compact invariant global attractor of system (1) in $C$ under some of the conditions of theorem 2.2.

Lemma 3.1. Assume that $f \in C\left(C, \mathbb{R}^{N}\right)$ and there exists $r \gg 0$ such that

$$
\forall i \in I_{N}, \forall x \in C \text { with } x_{i} \geqslant r_{i}, f_{i}(x)<0 .
$$

For the flow $\varphi_{\text {, }}$ generated by system (1) define the set

$$
A=\cap\left\{\varphi_{t}([0, r]): t \geqslant 0\right\} .
$$

Then A is nonempty, compact,

$$
A=\cup\{\gamma(x): x \in[0, r] \text { such that } \gamma(x) \text { is bounded }\} \text {, }
$$

and

$$
\forall t \in \mathbb{R}, \quad \varphi_{t}(A)=A .
$$

Proof. Clearly, $[0, r]$ is nonempty compact. From the assumptions we know that $[0, r]$ is positively invariant and globally attracting in $C$. So

$$
\forall t \geqslant 0, \quad \varphi_{t}([0, r]) \subset[0, r] .
$$

By continuity of $\varphi_{t}, \varphi_{t}([0, r])$ is nonempty compact for each $t \geqslant 0$. For any $t_{2}>t_{1}>0$, since $\varphi_{t_{2}-t_{1}}([0, r]) \subset[0, r]$, we must have

$$
\varphi_{t_{2}}([0, r])=\varphi_{t_{1}}\left(\varphi_{t_{2}-t_{1}}([0, r])\right) \subset \varphi_{t_{1}}([0, r]) \subset[0, r] .
$$

This shows that $\left\{\varphi_{t}([0, r])\right\}$ is a continuous decreasing sequence of nonempty compact sets. Thus, the set $A$ defined by (3) is a nonempty compact set.

We next show (4). From the assumptions we know that $\gamma^{-}(x)$ is unbounded for any $x \in C$ with $x_{i}>r_{i}$ for some $i \in I_{N}$. Thus, for each $x \in[0, r]$, if $\gamma(x)$ is bounded then we must have $\gamma(x) \subset[0, r]$. As $\varphi_{t}(\gamma(x))=\gamma(x)$ for all $t \in \mathbb{R}$, we obtain

$$
\forall t \geqslant 0, \quad \gamma(x)=\varphi_{t}(\gamma(x)) \subset \varphi_{t}([0, r])
$$

so $\gamma(x) \subset A$. If $\gamma(x)$ is unbounded then $\gamma(x) \not \subset[0, r]$, so there is a point $y \in \gamma(x) \backslash[0, r]$ such that $\gamma(x)=\gamma^{+}(y) \cup \gamma^{-}(y)$ with $\gamma^{-}(y) \subset C \backslash[0, r]$. For any $p \in \gamma^{+}(y)$, there is a $t \geqslant 0$ such that $p=\varphi_{t}(y)$. As $y \notin[0, r]$, by uniqueness of the solution, we have $p \notin \varphi_{t}([0, r])$ so $p \notin A$. This shows that $A \cap \gamma(x)=A \cap \gamma^{+}(y)=\emptyset$. Therefore, (4) holds.

Finally, the invariance of $A$, i.e. (5), follows from (4) and the invariance of any bounded orbit $\gamma(x)$.

Our next lemma further describes the monotone nature of negative half orbits in the global attractor $A$. 
Lemma 3.2. Assume that the conditions of theorem 2.2 are met. If $q \in A$ and $p \in[0, r]$ such that $p<q$, then, for each $i \in I_{N}$ with $p_{i}<q_{i}$, we have $\left(\varphi_{t}(p)\right)_{i}<\left(\varphi_{t}(q)\right)_{i}$ for all $t \leqslant 0$ and $\alpha(p) \subset \pi_{i}$.

Proof. Fix $i \in I_{N}$ such that $p_{i}<q_{i}$. If $p_{i}=0$, then $\left(\varphi_{t}(p)\right)_{i}=0<\left(\varphi_{t}(q)\right)_{i}$ for all $t \leqslant 0$ and $\gamma^{-}(p) \subset \pi_{i}$ so $\alpha(p) \subset \pi_{i}$.

Now suppose $0<p_{i}<q_{i}$. As $\varphi_{t}(p)$ and $\varphi_{t}(q)$ are solutions of (1) with $\varphi_{0}(p)=p$ and $\varphi_{0}(q)=q$ respectively, from (1) we have

$$
\begin{aligned}
& \left(\varphi_{t}(p)\right)_{i}=p_{i} \exp \left(\int_{0}^{t} f_{i}\left(\varphi_{s}(p)\right) \mathrm{d} s\right), \\
& \left(\varphi_{t}(q)\right)_{i}=q_{i} \exp \left(\int_{0}^{t} f_{i}\left(\varphi_{s}(q)\right) \mathrm{d} s\right),
\end{aligned}
$$

so

$$
\frac{\left(\varphi_{t}(q)\right)_{i}}{\left(\varphi_{t}(p)\right)_{i}}=\frac{q_{i}}{p_{i}} \exp \left(\int_{0}^{t}\left[f_{i}\left(\varphi_{s}(q)\right)-f_{i}\left(\varphi_{s}(p)\right)\right] \mathrm{d} s\right)
$$

For fixed $t<0$ and each $s \in[t, 0]$,

$$
\begin{aligned}
f_{i}\left(\varphi_{s}(q)\right)-f_{i}\left(\varphi_{s}(p)\right) & =\int_{0}^{1}\left[\frac{\mathrm{d}}{\mathrm{d} \theta} f_{i}\left(\varphi_{s}(p)+\theta\left(\varphi_{s}(q)-\varphi_{s}(p)\right)\right)\right] \mathrm{d} \theta \\
& =\sum_{j=1}^{N} \int_{0}^{1}\left[\frac{\partial f_{i}}{\partial x_{j}}\left(\varphi_{s}(p)+\theta\left(\varphi_{s}(q)-\varphi_{s}(p)\right)\right)\right] \mathrm{d} \theta\left[\left(\varphi_{s}(q)\right)_{j}-\left(\varphi_{s}(p)\right)_{j}\right] .
\end{aligned}
$$

Since $p<q$, by conditions (b) and (c) in theorem 2.2, the dynamics of (1) are monotone for $t \leqslant 0$ so $\varphi_{t}(p)<\varphi_{t}(q)$ for all $t<0$. Thus, by (c) again,

$$
f_{i}\left(\varphi_{s}(q)\right)-f_{i}\left(\varphi_{s}(p)\right) \leqslant \int_{0}^{1}\left[\frac{\partial f_{i}}{\partial x_{i}}\left(\varphi_{s}(p)+\theta\left(\varphi_{s}(q)-\varphi_{s}(p)\right)\right)\right] \mathrm{d} \theta \times\left[\left(\varphi_{s}(q)\right)_{i}-\left(\varphi_{s}(p)\right)_{i}\right] .
$$

Let

$$
m_{i}=-\max \left\{\frac{\partial f_{i}(x)}{\partial x_{i}}: x \in[0, r]\right\}
$$

By (c) we have $m_{i}>0$. As $\gamma(q) \subset A \subset[0, r]$ and $\varphi_{t}(p)<\varphi_{t}(q)$ for all $t \leqslant 0$, we also have $\gamma^{-}(p) \subset[0, r]$. Then the above inequality can be further simplified as

$$
f_{i}\left(\varphi_{s}(q)\right)-f_{i}\left(\varphi_{s}(p)\right) \leqslant-m_{i}\left[\left(\varphi_{s}(q)\right)_{i}-\left(\varphi_{s}(p)\right)_{i}\right] .
$$

Then, from (6) and (7) we obtain

$$
\frac{\left(\varphi_{t}(q)\right)_{i}}{\left(\varphi_{t}(p)\right)_{i}} \geqslant \frac{q_{i}}{p_{i}} \exp \left(m_{i} \int_{t}^{0}\left[\left(\varphi_{s}(q)\right)_{i}-\left(\varphi_{s}(p)\right)_{i}\right] \mathrm{d} s\right), \quad t \leqslant 0 .
$$

As $p_{i}<q_{i}$ and $\varphi_{t}(p)<\varphi_{t}(q)$ for all $t \leqslant 0$, we have

$$
\exp \left(m_{i} \int_{t}^{0}\left[\left(\varphi_{s}(q)\right)_{i}-\left(\varphi_{s}(p)\right)_{i}\right] \mathrm{d} s\right) \geqslant 1, \quad t<0 .
$$


It then follows from (8) that

$$
\frac{\left(\varphi_{t}(q)\right)_{i}}{\left(\varphi_{t}(p)\right)_{i}} \geqslant \frac{q_{i}}{p_{i}}>1, \quad t<0
$$

Thus,

$$
\left(\varphi_{t}(q)\right)_{i}-\left(\varphi_{t}(p)\right)_{i} \geqslant\left(\frac{q_{i}}{p_{i}}-1\right)\left(\varphi_{t}(p)\right)_{i}>0, t<0
$$

From (8) and (9) we obtain

$$
\frac{\left(\varphi_{t}(q)\right)_{i}}{\left(\varphi_{t}(p)\right)_{i}} \geqslant \frac{q_{i}}{p_{i}} \exp \left[m_{i}\left(\frac{q_{i}}{p_{i}}-1\right) \int_{t}^{0}\left(\varphi_{s}(p)\right)_{i} \mathrm{~d} s\right], \quad t \leqslant 0 .
$$

Finally, we are in a position to show by contradiction that

$$
\lim _{t \rightarrow-\infty}\left(\varphi_{t}(p)\right)_{i}=0
$$

Suppose (11) is false. Then there is an $\eta \in(0,1)$ and a sequence $\left\{t_{n}\right\} \subset(-\infty,-1)$ with $t_{n+1}<$ $t_{n}-1$ such that $\left(\varphi_{t_{n}}(p)\right)_{i} \geqslant \eta$ for all $n \geqslant 1$. Let

$$
M_{i}=\max \left\{x_{i}\left|f_{i}(x)\right|: x \in[0, r]\right\}+4
$$

and define a function $g:(-\infty, 0] \rightarrow \mathbb{R}_{+}$as follows:

$$
\forall n \geqslant 1, \forall t \in\left[t_{n}-\frac{\eta}{M_{i}}, t_{n}+\frac{\eta}{M_{i}}\right], \quad g(t)=\eta-M_{i}\left|t-t_{n}\right|,
$$

and $g(t)=0$ for $t \in(-\infty, 0] \backslash\left(\cup_{n=1}^{\infty}\left[t_{n}-\frac{\eta}{M_{i}}, t_{n}+\frac{\eta}{M_{i}}\right]\right)$. We claim that $\left(\varphi_{t}(p)\right)_{i} \geqslant g(t)$ for all $t \leqslant 0$. Indeed, for $t \in\left[t_{n}-\frac{\eta}{M_{i}}, t_{n}+\frac{\eta}{M_{i}}\right]$,

$$
\left|\left(\varphi_{t}(p)\right)_{i}-\left(\varphi_{t_{n}}(p)\right)_{i}\right| \leqslant\left|\int_{t_{n}}^{t}\left(\varphi_{s}(p)\right)_{i} f_{i}\left(\varphi_{s}(p)\right) \mathrm{d} s\right| \leqslant M_{i}\left|t-t_{n}\right|,
$$

so

$$
\left(\varphi_{t}(p)\right)_{i} \geqslant\left(\varphi_{t_{n}}(p)\right)_{i}-M_{i}\left|t-t_{n}\right| \geqslant g(t)
$$

As

$$
\int_{t_{n}-\frac{\eta}{M_{i}}}^{t_{n}+\frac{\eta}{M_{i}}} g(t) \mathrm{d} t=\frac{\eta^{2}}{M_{i}}>0
$$

we have

$$
\int_{t}^{0}\left(\varphi_{s}(p)\right)_{i} \mathrm{~d} s \geqslant \int_{t}^{0} g(s) \mathrm{d} s \rightarrow+\infty \quad(t \rightarrow-\infty) .
$$

From (10) we obtain $\frac{\left(\varphi_{t}(q)\right)_{i}}{\left(\varphi_{t}(p)\right)_{i}} \rightarrow+\infty$ as $t \rightarrow-\infty$. In particular, $\frac{\left(\varphi_{t_{n}}(q)\right)_{i}}{\left(\varphi_{t_{n}}(p)\right)_{i}} \rightarrow+\infty$ as $n \rightarrow \infty$. Since $\left(\varphi_{t_{n}}(p)\right)_{i} \geqslant \eta$ so $\frac{\left(\varphi_{t_{n}}(q)\right)_{i}}{\left(\varphi_{t_{n}}(p)\right)_{i}} \leqslant \frac{1}{\eta}\left(\varphi_{t_{n}}(q)\right)_{i}$, we must have $\left(\varphi_{t_{n}}(q)\right)_{i} \rightarrow+\infty$ as $n \rightarrow \infty$, a contradiction to the boundedness of $\gamma(q)$. Therefore, we must have (11) and thus $\alpha(p) \subset \pi_{i}$.

The next lemma shows that if the corresponding points on two positive half orbits are ordered pairs for all $t \geqslant 0$ then the two positive half orbits have the same positive limit set. 
Lemma 3.3. Assume that the conditions of theorem 2.2 are fulfilled. If for some $I \subset I_{N}$ and $x, y \in \dot{C}_{I}$, we have $\varphi_{t}(x) \leqslant \varphi_{t}(y)$ for all $t \geqslant 0$, then

$$
\lim _{t \rightarrow+\infty}\left(\varphi_{t}(y)-\varphi_{t}(x)\right)=0 .
$$

Proof. From (1) we see that $x, y \in \dot{C}_{I}$ implies $\varphi_{t}(x), \varphi_{t}(y) \in \dot{C}_{I}$ for all $t \geqslant 0$. By (b), $\varphi_{t}(x), \varphi_{t}(y) \in[0, r]$ for all large enough $t$. Without loss of generality, we may assume that $\varphi_{t}(x), \varphi_{t}(y) \in[0, r]$ for all $t \geqslant 0$. If $x=y$ the (13) is obviously true. Suppose $x<y$. Since $\varphi_{t}(x) \leqslant \varphi_{t}(y)$ for all $t \geqslant 0$, by uniqueness of the solution to the initial value problem of (1) we have $\varphi_{t}(x)<\varphi_{t}(y)$ for all $t \geqslant 0$.

For each fixed $t_{1}>0$ and every $i \in I$, if $\left(\varphi_{t_{1}}(x)\right)_{i}<\left(\varphi_{t_{1}}(y)\right)_{i}$ then by the same reasoning as that used in the proof of lemma 3.2, we have $\left(\varphi_{t}(x)\right)_{i}<\left(\varphi_{t}(y)\right)_{i}$ for all $t \in\left[0, t_{1}\right]$. Hence, if $\left(\varphi_{t_{1}}(x)\right)_{i}=\left(\varphi_{t_{1}}(y)\right)_{i}$, we must have $\left(\varphi_{t}(x)\right)_{i}=\left(\varphi_{t}(y)\right)_{i}$ for all $t \geqslant t_{1}$ so

$$
\lim _{t \rightarrow+\infty}\left[\left(\varphi_{t}(x)\right)_{i}-\left(\varphi_{t}(y)\right)_{i}\right]=0 .
$$

Now we show (14) under the assumption that $0<\left(\varphi_{t}(x)\right)_{i}<\left(\varphi_{t}(y)\right)_{i}$ for all $t \geqslant 0$. From (1) and (6) we have

$$
\frac{\left(\varphi_{t}(y)\right)_{i}}{\left(\varphi_{t}(x)\right)_{i}}=\frac{y_{i}}{x_{i}} \exp \left(\int_{0}^{t}\left[f_{i}\left(\varphi_{s}(y)\right)-f_{i}\left(\varphi_{s}(x)\right)\right] \mathrm{d} s\right), \quad t \geqslant 0 .
$$

From (15) and following the same lines between (6) and (8) in the proof of lemma 3.2 we obtain

$$
\frac{\left(\varphi_{t}(y)\right)_{i}}{\left(\varphi_{t}(x)\right)_{i}} \leqslant \frac{y_{i}}{x_{i}} \exp \left(-m_{i} \int_{0}^{t}\left[\left(\varphi_{s}(y)\right)_{i}-\left(\varphi_{s}(x)\right)_{i}\right] \mathrm{d} s\right), \quad t \geqslant 0 .
$$

Suppose (14) is false. Then there is a positive sequence $\left\{t_{n}\right\}$ satisfying $2<t_{n}+1<t_{n+1}$ and a $\delta \in(0,1)$ such that

$$
\left(\varphi_{t_{n}}(y)\right)_{i}-\left(\varphi_{t_{n}}(y)\right)_{i} \geqslant \delta, \quad \forall n \geqslant 1
$$

For $M_{i}$ defined by (12) and $t \in\left[t_{n}-\frac{\delta}{2 M_{i}}, t_{n}+\frac{\delta}{2 M_{i}}\right]$,

$$
\begin{aligned}
\left|\left[\left(\varphi_{t}(y)\right)_{i}-\left(\varphi_{t}(x)\right)_{i}\right]-\left[\left(\varphi_{t_{n}}(y)\right)_{i}-\left(\varphi_{t_{n}}(x)\right)_{i}\right]\right| \\
=\left|\int_{t_{n}}^{t}\left[\left(\varphi_{s}(y)\right)_{i} f_{i}\left(\varphi_{s}(y)\right)-\left(\varphi_{s}(x)\right)_{i} f_{i}\left(\varphi_{s}(x)\right)\right] \mathrm{d} s\right| \\
\quad \leqslant 2 M_{i}\left|t-t_{n}\right|
\end{aligned}
$$

so

$$
\begin{aligned}
\left(\varphi_{t}(y)\right)_{i}-\left(\varphi_{t}(x)\right)_{i} & \geqslant\left[\left(\varphi_{t_{n}}(y)\right)_{i}-\left(\varphi_{t_{n}}(x)\right)_{i}\right]-2 M_{i}\left|t-t_{n}\right| \\
& \geqslant \delta-2 M_{i}\left|t-t_{n}\right| .
\end{aligned}
$$

Then $\left(\varphi_{t}(y)\right)_{i}-\left(\varphi_{t}(x)\right)_{i} \geqslant h(t)$ for $t \geqslant 0$, where

$$
\forall n \geqslant 1, \forall t \in\left[t_{n}-\frac{\delta}{2 M_{i}}, t_{n}+\frac{\delta}{2 M_{i}}\right], \quad h(t)=\delta-2 M_{i}\left|t-t_{n}\right|
$$


and

$$
\forall t \in \mathbb{R}_{+} \backslash\left(\bigcup_{n=1}^{\infty}\left[t_{n}-\frac{\delta}{2 M_{i}}, t_{n}+\frac{\delta}{2 M_{i}}\right]\right), \quad h(t)=0 .
$$

As

$$
\int_{t_{n}-\frac{\delta}{2 M_{i}}}^{t_{n}+\frac{\delta}{2 M_{i}}} h(t) \mathrm{d} t=\frac{\delta^{2}}{2 M_{i}}>0
$$

we have

$$
\int_{0}^{t}\left[\left(\varphi_{s}(y)\right)_{i}-\left(\varphi_{s}(x)\right)_{i}\right] \mathrm{d} s \geqslant \int_{0}^{t} h(s) \mathrm{d} s \rightarrow+\infty \quad(t \rightarrow+\infty) .
$$

This together with (16) implies that

$$
\lim _{t \rightarrow+\infty} \frac{\left(\varphi_{t}(y)\right)_{i}}{\left(\varphi_{t}(x)\right)_{i}}=0
$$

In particular, we have

$$
\lim _{n \rightarrow \infty} \frac{\left(\varphi_{t_{n}}(y)\right)_{i}}{\left(\varphi_{t_{n}}(x)\right)_{i}}=0
$$

As $\left(\varphi_{t_{n}}(x)\right)_{i} \leqslant r_{i}$ so $\frac{\left(\varphi_{\left.t_{t_{n}}(y)\right)_{i}}\left(\varphi_{t_{n}}(x)\right)_{i}\right.}{r_{i}}\left(\varphi_{t_{n}}(y)\right)_{i}$, we must have $\lim _{n \rightarrow \infty}\left(\varphi_{t_{n}}(y)\right)_{i}=0$. Thus, there is $n_{1} \geqslant 1$ such that $\left(\varphi_{t_{n}}(y)\right)_{i} \leqslant \frac{1}{2} \delta$ for all $n \geqslant n_{1}$. Since $\left(\varphi_{t_{n}}(y)\right)_{i}-\left(\varphi_{t_{n}}(x)\right)_{i} \geqslant \delta$ for all $n \geqslant 1$, for $n \geqslant n_{1}$ we have

$$
\left(\varphi_{t_{n}}(x)\right)_{i} \leqslant\left(\varphi_{t_{n}}(y)\right)_{i}-\delta<\frac{1}{2} \delta-\delta=-\frac{1}{2} \delta<0,
$$

a contradiction to $\varphi_{t}(x) \in[0, r]$. This contradiction shows the truth of (14). Then (13) follows from (14) for all $i \in I$.

To prove theorem 2.2 we need to find a candidate for the required carrying simplex. By condition (a), the origin is a repellor. Let $\mathcal{B}(0)$ be the basin of repulsion of 0 in $C$. Then $\mathcal{B}(0)$ is open relative to $C$ and invariant. By condition (b), we can view $\infty$ as a repellor with $C \backslash[0, r] \subset \mathcal{B}(\infty)$. Thus, we must have $\mathcal{B}(0) \subset[0, r]$. For each $x \in \mathcal{B}(0), \gamma(x) \subset \mathcal{B}(0)$ so $\gamma(x)$ is bounded. From lemma 3.1 we have $\mathcal{B}(0) \subset A$. Let $\Sigma=A \backslash \mathcal{B}(0)$. Then $\Sigma$ is nonempty, compact and invariant. We are now able to show that $\Sigma$ is the required carrying simplex and to give the proof of theorem 2.2.

Proof of Theorem 2.2. We know from $\Sigma=A \backslash \mathcal{B}(0)$ that $\Sigma$ is an invariant submanifold.

Firstly, we show that $\Sigma$ is homeomorphic to $\Delta^{N-1}$ by radial projection. For each $x \in \Delta^{N-1}$, there is a $\lambda_{0}=\lambda_{0}(x)>0$ such that $\lambda_{0} x \in A$ but $\lambda x \notin A$ for all $\lambda>\lambda_{0}$. For each $\lambda \in\left(0, \lambda_{0}\right)$, since $\lambda x<\lambda_{0} x$, by lemma 3.2 we have $\lim _{t \rightarrow-\infty} \varphi_{t}(\lambda x)=0$, so $\lambda x \in \mathcal{B}(0) \subset A$ for all $\lambda \in$ $\left[0, \lambda_{0}\right)$. As $\mathcal{B}(0)$ is open but $A$ is closed, we must have $\lambda_{0} x \in A \backslash \mathcal{B}(0)=\Sigma$. Thus, for each $x \in \Delta^{N-1}$ there is a unique $\lambda_{0}(x)>0$ such that $\lambda_{0}(x) x \in \Sigma$. Conversely, for each $y \in \Sigma$, there is a unique $\mu_{0}(y)>0$ such that $\mu_{0}(y) y \in \Delta^{N-1}$.

Next, we need to show that both $\lambda_{0}(x)$ and $\mu_{0}(y)$ are continuous. Suppose $\lambda_{0}(x)$ is not continuous on $\Delta^{N-1}$. Then there exist a sequence $\left\{x_{(n)}\right\} \subset \Delta^{N-1}$, a point $p \in \Delta^{N-1}$ and a number $\lambda_{1}>0$ such that, as $n \rightarrow \infty$,

$$
x_{(n)} \rightarrow p, \quad \lambda_{0}\left(x_{(n)}\right) \rightarrow \lambda_{1} \neq \lambda_{0}(p),
$$

so that 


$$
\lambda_{0}\left(x_{(n)}\right) x_{(n)} \rightarrow \lambda_{1} p \neq \lambda_{0}(p) p .
$$

Since $\lambda_{0}(p) p \in \Sigma$ and $\lambda_{0}\left(x_{(n)}\right) x_{(n)} \in \Sigma$ for all $n \geqslant 1$, by the compactness of $\Sigma$ we have $\lambda_{1} p \in \Sigma$. As $\lambda_{1} \neq \lambda_{0}(p)$, we have either $\lambda_{1} p<\lambda_{0}(p) p$ or $\lambda_{0}(p) p<\lambda_{1} p$. By lemma 3.2, we have either $\lambda_{1} p \in \mathcal{B}(0)$ or $\lambda_{0}(p) p \in \mathcal{B}(0)$, a contradiction to $\lambda_{1} p \in \Sigma$ and $\lambda_{0}(p) p \in \Sigma$. This shows the continuity of $\lambda_{0}(x)$. The continuity of $\mu_{0}(y)$ follows similarly. Therefore, $\Sigma$ is homeomorphic to $\Delta^{N-1}$ by radial projection.

Secondly, we prove that for each $x \in C \backslash\{0\}$, there is a $y \in \Sigma$ such that

$$
\lim _{t \rightarrow+\infty}\left[\varphi_{t}(x)-\varphi_{t}(y)\right]=0 .
$$

If $x \in \Sigma$ then we take $y=x$ and (17) holds obviously.

Now suppose $x \in C \backslash A$. If we can find a $y \in \Sigma$ such that $\varphi_{t}(y) \leqslant \varphi_{t}(x)$ for all $t \geqslant 0$, then (17) follows from lemma 3.3. We now show that such a $y$ exists. By (b) there is a $T>0$ such that $\varphi_{t}(x) \in[0, r]$ for all $t \geqslant T$. Without loss of generality, we suppose $x \in[0, r] \backslash A$ so that $\varphi_{t}(x) \in[0, r] \backslash A$ for all $t \geqslant 0$. For this $x$ and each fixed $t \geqslant 0$, define the set

$$
S(t, x)=\left\{y \in \Sigma: \varphi_{t}(y) \leqslant \varphi_{t}(x)\right\} .
$$

We need to check that $S(t, x) \neq \emptyset$. As $\varphi_{s}(A)=A$ for all $s \in \mathbb{R}$ by lemma 3.1 but $\varphi_{t}(x) \in$ $[0, r] \backslash A$, there is an $\eta_{0} \in(0,1)$ such that $\eta_{0} \varphi_{t}(x) \in \Sigma$ but $\eta \varphi_{t}(x) \notin \Sigma$ for $\eta_{0}<\eta \leqslant 1$. As $\Sigma$ is invariant, taking $y=\varphi_{-t}\left(\eta_{0} \varphi_{t}(x)\right)$ we have $y \in \Sigma$ and

$$
\varphi_{t}(y)=\eta_{0} \varphi_{t}(x)<\varphi_{t}(x) .
$$

Thus, $\varphi_{-t}\left(\eta_{0} \varphi_{t}(x)\right) \in S(t, x)$ and $S(t, x) \neq \emptyset$.

For any $t_{2}>t_{1} \geqslant 0$, if $y \in S\left(t_{2}, x\right)$ then $\varphi_{t_{2}}(y)<\varphi_{t_{2}}(x)$. By the monotone property for competitive systems, we have

$$
\varphi_{t}(y)=\varphi_{t-t_{2}}\left(\varphi_{t_{2}}(y)\right)<\varphi_{t-t_{2}}\left(\varphi_{t_{2}}(x)\right)=\varphi_{t}(x)
$$

for all $t \in\left[0, t_{2}\right]$. In particular, $\varphi_{t_{1}}(y)<\varphi_{t_{1}}(x)$. Thus, $y \in S\left(t_{1}, x\right)$. This shows that

$$
\forall t_{2}>t_{1} \geqslant 0, \quad S\left(t_{2}, x\right) \subset S\left(t_{1}, x\right) .
$$

From the definition we see that each $S(t, x)$ is compact. Hence, $\cap_{t \geqslant 0} S(t, x) \neq \emptyset$. Then, for each $y \in \cap_{t \geqslant 0} S(t, x) \subset \Sigma$ and all $t \geqslant 0$, we have $\varphi_{t}(y) \leqslant \varphi_{t}(x)$, so (17) follows from lemma 3.3.

Next, we suppose $x \in \mathcal{B}(0) \backslash\{0\}$ and show the existence of $y \in \Sigma$ such that $\varphi_{t}(x) \leqslant \varphi_{t}(y)$ for all $t \geqslant 0$. For each $t \geqslant 0$, define a set

$$
U(t, x)=\left\{y \in \Sigma: \varphi_{t}(x) \leqslant \varphi_{t}(y)\right\} .
$$

Using the techniques similar to those in the above paragraph we can check that $U(t, x)$ is nonempty, compact and

$$
\forall t_{2}>t_{1} \geqslant 0, \quad U\left(t_{2}, x\right) \subset U\left(t_{1}, x\right) .
$$

Hence, $\cap_{t} \geqslant 0 U(t, x) \neq \emptyset$. Now for any $y \in \cap_{t \geqslant 0} U(t, x)$, we have $\varphi_{t}(x) \leqslant \varphi_{t}(y)$ for all $t \geqslant 0$. Then (17) follows from lemma 3.3. So far we have proved that $\Sigma$ is a carrying simplex.

Thirdly, we show that $\Sigma$ is the unique carrying simplex. Suppose (1) has another carrying simplex $\Sigma_{1} \neq \Sigma$. Then there exist a point $p \in \Delta^{N-1}$ and two distinct numbers $\lambda_{0}>0$ and $\lambda_{1}>0$ such that $\lambda_{0} p \in \Sigma$ and $\lambda_{1} p \in \Sigma_{1}$. If $\lambda_{1}<\lambda_{0}$ then $\lambda_{1} p<\lambda_{0} p$ so $\lambda_{1} p \in \mathcal{B}(0)$ by lemma 3.2. As $\gamma\left(\lambda_{1} p\right) \subset \Sigma_{1}$ and $\Sigma_{1}$ is compact, we must have $\{0\}=\alpha\left(\lambda_{1} p\right) \subset \Sigma_{1}$, a contradiction to 
$\Sigma_{1}$ being homeomorphic to $\Delta^{N-1}$ by radial projection. If $\lambda_{1}>\lambda_{0}$ then $\lambda_{1} p>\lambda_{0} p$ so $\lambda_{1} p \notin A$ and $\gamma\left(\lambda_{1} p\right)$ is unbounded, a contradiction to the boundedness of $\Sigma_{1}$ as $\gamma\left(\lambda_{1} p\right) \subset \Sigma_{1}$. These contradictions show that $\Sigma$ is the unique carrying simplex of (1).

Finally, for each $p \in \Sigma$ and every $q \in[0, r] \backslash\{0\}$ with $q<p$, by lemma 3.2, for each $i \in I_{N}$ with $q_{i}<p_{i}$ we have $\alpha(q) \subset \pi_{i}$.

Next, to prove corollary 2.4, we define a set $S \subset \mathbb{R}^{N}$ to be order convex if any $x, y \in S$ with $x<y$ implies $[x, y] \subset S$. A point $z$ is defined to be in the upper boundary of a set $S$ if there is a sequence $s_{i}$ in $S$ converging to $z$ such that $s_{i} \ll z$ for all $i$ but no sequence $x_{i}$ in $S$ converging to $z$ such that $x_{i} \gg z$ for all $i$.

Proof of Corollary 2.4. We know that $\Sigma=A \backslash \mathcal{B}(0)$, where $A$ defined in lemma 3.1 is compact invariant. As $\mathcal{B}(0) \subset A$ is open in the space $C$ and $\Sigma$ is homeomorphic to $\Delta^{N-1}$ by radial projection, we see that $A$ is equal to the closure of $\mathcal{B}(0)$. We now claim that $A$ is order convex. Indeed, for any $p \in \Sigma$, there is an $I \subset I_{N}$ such that $p \gg_{I} 0$. By theorem 2.2, we have $(0, p) \subset \mathcal{B}(0)$. Taking closure on both sides, we obtain $[0, p] \subset A$. Then, for any $a, b \in A$ with $a<b$, there is a $\lambda \geqslant 1$ such that $\lambda b \in \Sigma$. so $[a, b] \subset[0, \lambda b] \subset A$. Thus, $A$ is order convex.

Clearly, int $\Sigma=\Sigma \cap \dot{C}$ is the upper boundary of $A$. Then, by proposition 2.6 in [9] (its proof is still valid here), the map $g: \operatorname{int} \Sigma \rightarrow E$ defined by the orthogonal projection $P$ restricted to int $\Sigma$ is a Lipschitz homeomorphism, $g\left(\right.$ int $\Sigma$ ) is an open set of $E$, and $g^{-1}$ is also Lipschitz. Then extending the definition of $g$ to $\Sigma$ naturally, so $g^{-1}: g(\Sigma) \rightarrow \Sigma, g$ is still a homeomorphism with both $g$ and $g^{-1}$ Lipschitz.

For any $x \in g(\Sigma)$, define the flow $\theta_{t}$ through $x$ by

$$
\theta_{t}(x)=g \circ \varphi_{t} \circ g^{-1}(x)=g\left(\varphi_{t}\left(g^{-1}(x)\right)\right) .
$$

As $\Sigma$ is invariant under the flow $\varphi_{t}$ and $g^{-1}(x) \in \Sigma, \varphi_{t}\left(g^{-1}(x)\right) \in \Sigma$ for all $t \in \mathbb{R}$. Thus, $\theta_{t}(x) \in$ $g(\Sigma)$ for all $t \in \mathbb{R}$ and

$$
\theta_{t}(x)=\varphi_{t}\left(g^{-1}(x)\right)-\left(u \cdot \varphi_{t}\left(g^{-1}(x)\right)\right) u .
$$

Then

$$
\begin{aligned}
\frac{\mathrm{d}}{\mathrm{d} t}\left(\theta_{t}(x)\right) & =\frac{\mathrm{d}}{\mathrm{d} t}\left(\varphi_{t}\left(g^{-1}(x)\right)\right)-\left(u \cdot \frac{\mathrm{d}}{\mathrm{d} t}\left(\varphi_{t}\left(g^{-1}(x)\right)\right)\right) u \\
& =F\left(\varphi_{t}\left(g^{-1}(x)\right)\right)-\left(u \cdot F\left(\varphi_{t}\left(g^{-1}(x)\right)\right)\right) u \\
& =P \circ F \circ \varphi_{t} \circ g^{-1}(x) \\
& =P \circ F \circ g^{-1} \circ\left(g \circ \varphi_{t} \circ g^{-1}(x)\right) \\
& =P \circ F \circ g^{-1}\left(\theta_{t}(x)\right) .
\end{aligned}
$$

Thus, the flow $\theta_{t}$ on $g(\Sigma) \subset E$ is conjugate to the flow $\varphi_{t}$ on $\Sigma$ and it is determined by the vector field $G(y)=P \circ F \circ g^{-1}(y)$. Clearly, $G$ is Lipschitz as $P, F$ and $g^{-1}$ are Lipschitz.

\section{Heteroclinic limit cycles in three-dimensional systems}

In this section, we consider competitive system (1) with $N=3$ and present a result on heteroclinic limit cycles as an application of theorem 2.2, which is an update of a result based 
on theorem 1.1 with a stronger assumption that (1) is totally competitive [19]. ('Strongly competitive' was used there for the same meaning as totally competitive here.) We assume that the conditions of theorem 2.2 are satisfied so that (1) has a unique carrying simplex $\Sigma$.

By a heteroclinic cycle we mean a closed curve that is topologically a circle consisting of fixed points $p_{(i)}$ for $i \in I_{3}$, together with heteroclinic trajectories $T_{i}$ connecting $p_{(i)}$ to $p_{(i+1)}$ (here $\left.p_{(4)}=p_{(1)}\right)$. By a heteroclinic limit cycle $\Gamma$ we mean a heteroclinic cycle $\Gamma$ with an attracting (or repelling) neighbourhood $N(\Gamma)$ (restricted to $\dot{C}$ or $C$ ) such that $\omega\left(x^{0}\right)=\Gamma$ (or $\left.\alpha\left(x^{0}\right)=\Gamma\right)$ for all $x^{0} \in N(\Gamma)$. The main issue we address here is when the heteroclinic cycle is globally attracting or repelling (in some sense defined below).

Definition 4.1. We say that a heteroclinic cycle $\Gamma_{0}$ of (1) is a

- locally attracting (repelling) heteroclinic limit cycle if there is a neighbourhood $V \subset \dot{C}$ $(V \subset$ int $\Sigma)$ of $\Gamma_{0}$ such that $\omega\left(x^{0}\right)=\Gamma_{0}\left(\alpha\left(x^{0}\right)=\Gamma_{0}\right)$ for all $x^{0} \in V$;

- globally attracting (repelling) heteroclinic limit cycle if $\omega\left(x^{0}\right)=\Gamma_{0}\left(\alpha\left(x^{0}\right)=\Gamma_{0}\right)$ for all $x^{0} \in \dot{C} \backslash U\left(x^{0} \in \operatorname{int} \Sigma \backslash U\right)$, where $U$ is a union of a finite number of manifolds of dimension lower than 3 (2) and the set of fixed points.

The theorem below is an update of theorem 2.3 in [19]. The proof of theorem 2.3 given in [19] is still valid for theorem 4.2.

Theorem 4.2. In addition to the conditions of theorem 2.2, we assume that the Kolmogorov system (1) with $N=3$ satisfies the following conditions $(a)$ and $(b)$ or $(c)$ as well:

(a) The three axial fixed points $Q_{1}, Q_{2}, Q_{3}$ are the only fixed points of (1) on $\partial \Sigma$ and either the inequalities (18) or (19) hold:

$$
\begin{aligned}
& \forall i \in I_{3}, \quad f_{i}\left(Q_{i+1}\right)<0<f_{i+2}\left(Q_{i+1}\right), \\
& \forall i \in I_{3}, \quad f_{i}\left(Q_{i+1}\right)>0>f_{i+2}\left(Q_{i+1}\right) .
\end{aligned}
$$

(b) There is a unique fixed point $p$ in int $\Sigma$ that is globally asymptotically stable.

(c) There is a unique fixed point $p$ in int $\Sigma$ that is hyperbolic with one-dimensional stable manifold $W^{s}(p)$ in $\dot{C}$ and globally repelling on $\Sigma$.

Then, under $(a)$ and $(b), \partial \Sigma$ is a heteroclinic limit cycle globally repelling on $\Sigma: \alpha\left(x^{0}\right)=$ $\partial \Sigma$ for all $x^{0} \in \operatorname{int} \Sigma \backslash\{p\} ;$ under $(a)$ and $(c), \partial \Sigma$ is a heteroclinic limit cycle globally attracting in $\dot{C}: \omega\left(x^{0}\right)=\partial \Sigma$ for all $x^{0} \in \dot{C} \backslash W^{s}(p)$.

In the rest of this section, we analyse two concrete systems as examples to demonstrate the application of theorems 2.2 and 4.2. Without theorem 2.2 it is not possible to deal with the two systems as theorem 1.1 is not applicable to them due to some zero off-diagonal entries in $D f$.

For any vector $a=\left(a_{1}, a_{2}, a_{3}\right)>0, C$ has the partition $C=C_{-} \cup \Pi \cup C_{+}$by the plane $\Pi=\left\{x \in C: a_{1} x_{1}+a_{2} x_{2}+a_{3} x_{3}=1\right\}$ restricted to $C$, where $0 \in C_{-}$. A point $p \in C$ is said to be below $\Pi$ if $p \in C_{-}$, above $\Pi$ if $p \in C_{+}$, and on $\Pi$ if $p \in \Pi$. A nonempty set $S \subset C$ is said to be strictly below $\Pi$ if $S \subset C_{-}$and strictly above $\Pi$ if $S \subset C_{+}$.

Example 1. Consider the three-dimensional system

$$
\begin{aligned}
& \dot{x}_{1}=x_{1} f_{1}(x) \equiv x_{1}\left[1-x_{1}-(2-\alpha) x_{2}-\alpha\left(2 x_{1}+x_{2}\right) x_{2}\right], \\
& \dot{x}_{2}=x_{2} f_{2}(x) \equiv x_{2}\left[1-x_{2}-(2-\alpha) x_{3}-\alpha\left(2 x_{2}+x_{3}\right) x_{3}\right], \\
& \dot{x}_{3}=x_{3} f_{3}(x) \equiv x_{3}\left[1-x_{3}-(2-\alpha) x_{1}-\alpha\left(2 x_{3}+x_{1}\right) x_{1}\right],
\end{aligned}
$$


where $\alpha \in(0,2)$ is a parameter. Take $r_{1}=r_{2}=r_{3}=1.1$. Then (20) satisfies the conditions of theorem 2.2 so it has a unique carrying simplex $\Sigma$.

Proposition 4.3. System (20) with $\alpha \in(0,2)$ has a heteroclinic limit cycle $\partial \Sigma$ globally repelling on $\Sigma$.

Proof. Clearly, (20) has three axial equilibria $e_{1}=(1,0,0), e_{2}=(0,1,0)$ and $e_{3}=(0,0,1)$ and an interior equilibrium $p=\left(\frac{1}{3}, \frac{1}{3}, \frac{1}{3}\right)$. Since $e_{1}, e_{2}, e_{3}$ are the only equilibria on $\partial \Sigma$ and

$$
\begin{aligned}
& f_{1}\left(e_{2}\right)=f_{2}\left(e_{3}\right)=f_{3}\left(e_{1}\right)=-1<0, \\
& f_{3}\left(e_{2}\right)=f_{1}\left(e_{3}\right)=f_{2}\left(e_{1}\right)=1>0,
\end{aligned}
$$

system (20) satisfies condition (a) of theorem 4.2. We shall show that $p$ is globally asymptotically stable in $\dot{C}$ so that the conclusion of proposition 4.3 follows from theorem 4.2.

Note that the half-line $L=\{k p: k>0\}$ is an invariant manifold satisfying $\lim _{t \rightarrow+\infty} \varphi_{t}(x)=p$ for all $x \in L$. To show that $p$ is globally attracting in $\dot{C}$, we let $V(x)=x_{1} x_{2} x_{3}$ and $W(x)=x_{1}+x_{2}+x_{3}$. Then

$$
\left.\dot{V}\right|_{(20)}=V(x)(3+\alpha W(x))(1-W(x))
$$

and

$$
\left.\dot{W}\right|_{(20)}=W(x)-W(x)^{2}+\alpha\left(x_{1} x_{2}+x_{2} x_{3}+x_{3} x_{1}-U\right),
$$

where

$$
U=2 x_{1}^{2} x_{2}+x_{1} x_{2}^{2}+2 x_{2}^{2} x_{3}+x_{2} x_{3}^{2}+2 x_{3}^{2} x_{1}+x_{3} x_{1}^{2}
$$

As

$$
W(x)\left(x_{1} x_{2}+x_{2} x_{3}+x_{3} x_{1}\right)=3 x_{1} x_{2} x_{3}+x_{1}^{2} x_{2}+x_{1} x_{2}^{2}+x_{1}^{2} x_{3}+x_{1} x_{3}^{2}+x_{2}^{2} x_{3}+x_{2} x_{3}^{2},
$$

the expression for $\left.\dot{W}\right|_{(20)}$ can be simplified as

$$
\left.\dot{W}\right|_{(20)}=(1-W)\left[W(x)+\alpha\left(x_{1} x_{2}+x_{2} x_{3}+x_{3} x_{1}\right)\right]-\alpha g\left(x_{1}, x_{2}, x_{3}\right),
$$

where

$$
g\left(x_{1}, x_{2}, x_{3}\right)=x_{1}^{2} x_{2}+x_{2}^{2} x_{3}+x_{3}^{2} x_{1}-3 x_{1} x_{2} x_{3} .
$$

Let

$$
\pi_{p}=\left\{x \in \mathbb{R}_{+}^{3}: x_{1}+x_{2}+x_{3}=1\right\}
$$

For $x \in \pi_{p}$ we have $W(x)=1$, so $\left.\dot{W}\right|_{(20)}=-\alpha g\left(x_{1}, x_{2}, x_{3}\right)$. If we can show that

$$
\forall x \in \pi_{p} \backslash\left\{e_{1}, e_{2}, e_{3}, p\right\}, g\left(x_{1}, x_{2}, x_{3}\right)>0,
$$

trajectories passing through $\pi_{p}$ will stay below $\pi_{p}$ forever. By the definition of $\Sigma$ we know that $\Sigma \backslash\left\{e_{1}, e_{2}, e_{3}, p\right\}$ is strictly below the plane $\pi_{p}$. From (21) we know that $\left.\dot{V}\right|_{(20)}>0$ for $x \in \operatorname{int} \Sigma \backslash\{p\}$. Thus, $\gamma^{+}(x) \subset$ int $\Sigma \backslash\{p\}$ and $V\left(\varphi_{t}(x)\right)$ is strictly increasing for $x \in \operatorname{int} \Sigma \backslash\{p\}$ and $t \in \mathbb{R}$. We claim that

$$
\forall x \in \text { int } \Sigma \backslash\{p\}, \lim _{t \rightarrow+\infty} \varphi_{t}(x)=p .
$$

Indeed, if (25) is not true then $\omega\left(x^{0}\right) \neq\{p\}$ for some $x^{0} \in \operatorname{int} \Sigma \backslash\{p\}$. As $V\left(\varphi_{t}\left(x^{0}\right)\right)$ is strictly increasing, there is a constant $c$ such that $V\left(\varphi_{t}\left(x^{0}\right)\right) \uparrow c$ as $t \rightarrow+\infty$. This implies that $\omega\left(x^{0}\right) \subset$ 
int $\Sigma$ and $V\left(\omega\left(x^{0}\right)\right)=c$. Since $\omega\left(x^{0}\right) \neq\{p\}, \omega\left(x^{0}\right) \backslash\{p\} \subset$ int $\Sigma \backslash\{p\}$ and int $\Sigma \backslash\{p\}$ is strictly below $\pi_{p}$, there is a $q \in \omega\left(x^{0}\right) \backslash\{p\}$ such that $\gamma(q) \subset \omega\left(x^{0}\right) \backslash\{p\}$ so that $\dot{V}\left(\varphi_{t}(q)\right)>0$. But this contradicts $V\left(\varphi_{t}(q)\right)=V\left(\omega\left(x^{0}\right)\right)=c$. Therefore, (25) holds and $p$ is globally attracting in $\dot{C}$. The Jacobian matrix at $p$ is

$$
J(p)=\left(\begin{array}{ccc}
-\frac{1}{9}(3+2 \alpha) & -\frac{1}{9}(6+\alpha) & 0 \\
0 & -\frac{1}{9}(3+2 \alpha) & -\frac{1}{9}(6+\alpha) \\
-\frac{1}{9}(6+\alpha) & 0 & -\frac{1}{9}(3+2 \alpha)
\end{array}\right)
$$

and $J(p)$ has eigenvalues

$$
\lambda_{1}=-1-\frac{1}{3} \alpha, \quad \lambda_{2,3}=-\frac{5}{18} \alpha \pm i \frac{\sqrt{3}}{18}(\alpha+6) .
$$

As $\operatorname{Re} \lambda_{j}<0$ for all $j \in I_{3}, p$ is globally asymptotically stable in the interior of $\mathbb{R}_{+}^{3}$.

Finally we show (24). Note that $x \in \pi_{p} \backslash\left\{e_{1}, e_{2}, e_{3}, p\right\}$ if and only if $x \in \pi_{p}$ with $\frac{1}{3}<x_{1}<1$ or $\frac{1}{3}<x_{2}<1$ or $\frac{1}{3}<x_{3}<1$. By rotational symmetry of $\pi_{p}$ and $g$, if $g\left(x_{1}, x_{2}, x_{3}\right)>0$ for $x \in \pi_{p}$ with $\frac{1}{3}<x_{2}<1$, then $g\left(x_{1}, x_{2}, x_{3}\right)>0$ for $x \in \pi_{p}$ with $\frac{1}{3}<x_{1}<1$ and $g\left(x_{1}, x_{2}, x_{3}\right)>0$ for $x \in \pi_{p}$ with $\frac{1}{3}<x_{3}<1$ so that (24) follows. Thus, by letting $G\left(x_{1}, x_{2}\right)=g\left(x_{1}, x_{2}, 1-x_{1}-x_{2}\right)$, we need only prove that

$$
\forall x_{2} \in(1 / 3,1), \forall x_{1} \in\left[0,1-x_{2}\right], \quad G\left(x_{1}, x_{2}\right)>0 .
$$

From the definition of $g$ we have

$$
\begin{aligned}
G\left(x_{1}, x_{2}\right) & =x_{1}^{2} x_{2}+\left(1-x_{1}-x_{2}\right)\left[x_{2}^{2}+\left(1-x_{1}-x_{2}\right) x_{1}-3 x_{1} x_{2}\right] \\
& =x_{1}^{3}+\left(6 x_{2}-2\right) x_{1}^{2}+\left(3 x_{2}^{2}-5 x_{2}+1\right) x_{1}+x_{2}^{2}-x_{2}^{3},
\end{aligned}
$$

so

$$
\begin{aligned}
\frac{\partial G}{\partial x_{1}} & =3 x_{1}^{2}+4\left(3 x_{2}-1\right) x_{1}+\left(3 x_{2}^{2}-5 x_{2}+1\right), \\
\frac{\partial^{2} G}{\partial x_{1}^{2}} & =6 x_{1}+4\left(3 x_{2}-1\right) .
\end{aligned}
$$

As $\frac{\partial^{2} G}{\partial x_{1}^{2}}>0$ for $x_{2}>\frac{1}{3}$ and $x_{1} \geqslant 0$, for each fixed $x_{2} \in\left(\frac{1}{3}, 1\right), \frac{\partial G}{\partial x_{1}}$ is strictly increasing for $x_{1} \in\left[0,1-x_{2}\right]$. If $x_{2} \in\left(\frac{5}{6}, 1\right)$ then

$$
\frac{\partial G}{\partial x_{1}}\left(x_{1}, x_{2}\right) \leqslant \frac{\partial G}{\partial x_{1}}\left(1-x_{2}, x_{2}\right)=-x_{2}\left(6 x_{2}-5\right)<0
$$

so $G\left(x_{1}, x_{2}\right)$ is strictly decreasing for $x_{1} \in\left[0,1-x_{2}\right]$, when $x_{2} \in\left[\frac{5}{6}, 1\right)$ is fixed, and

$$
G\left(x_{1}, x_{2}\right) \geqslant G\left(1-x_{2}, x_{2}\right)=\left(1-x_{2}\right)^{2} x_{2}>0 .
$$

For fixed $x_{2} \in\left(\frac{1}{3}, \frac{5}{6}\right)$, we have $\frac{\partial G}{\partial x_{1}}\left(1-x_{2}, x_{2}\right)>0$ but

$$
\frac{\partial G}{\partial x_{1}}\left(0, x_{2}\right)=3 x_{2}^{2}-5 x_{2}+1=3\left(x_{2}-\frac{5-\sqrt{13}}{6}\right)\left(x_{2}-\frac{5+\sqrt{13}}{6}\right)<0 \text {. }
$$


Thus, $\frac{\partial G}{\partial x_{1}}\left(x_{1}, x_{2}\right)=0$ at

$$
x_{1}^{*}=\frac{1}{6}\left(\sqrt{108\left(x_{2}-1 / 6\right)^{2}+1}-4\left(3 x_{2}-1\right)\right)
$$

so $G\left(x_{1}^{*}, x_{2}\right)$ is the minimum of $G\left(x_{1}, x_{2}\right)$ for $x_{1} \in\left[0,1-x_{2}\right]$. From (27) we have

$$
\begin{aligned}
\left(x_{1}^{*}\right)^{2}= & \frac{1}{9}\left[63 x_{2}^{2}-33 x_{2}+5-2\left(3 x_{2}-1\right) \sqrt{108\left(x_{2}-1 / 6\right)^{2}+1}\right] \\
\left(x_{1}^{*}\right)^{3}= & \frac{1}{54}\left[-36 \times 39 x_{2}^{3}+27 \times 40 x_{2}^{2}-288 x_{2}+28\right. \\
& \left.+\left(135 x_{2}^{2}-81 x_{2}+13\right) \sqrt{108\left(x_{2}-1 / 6\right)^{2}+1}\right] .
\end{aligned}
$$

Let $H\left(x_{2}\right)=G\left(x_{1}^{*}, x_{2}\right)$. Then, substitution of (27)-(29) into $H\left(x_{2}\right)$ and simplification gives

$$
H\left(x_{2}\right)=9 x_{2}^{3}-3 x_{2}^{2}+\frac{2}{27}-\frac{1}{108}\left[108\left(x_{2}-1 / 6\right)^{2}+1\right]^{\frac{3}{2}} .
$$

The derivatives of $H\left(x_{2}\right)$ are

$$
\begin{aligned}
& H^{\prime}\left(x_{2}\right)=27 x_{2}^{2}-6 x_{2}-3\left(x_{2}-1 / 6\right)\left[108\left(x_{2}-1 / 6\right)^{2}+1\right]^{\frac{1}{2}}, \\
& H^{\prime \prime}\left(x_{2}\right)=54 x_{2}-6-3\left[216\left(x_{2}-1 / 6\right)^{2}+1\right]\left[108\left(x_{2}-1 / 6\right)^{2}+1\right]^{-\frac{1}{2}}, \\
& H^{\prime \prime \prime}\left(x_{2}\right)=54-324\left(x_{2}-1 / 6\right)\left[216\left(x_{2}-1 / 6\right)^{2}+3\right]\left[108\left(x_{2}-1 / 6\right)^{2}+1\right]^{-\frac{3}{2}} .
\end{aligned}
$$

It can be verified that $H^{(4)}\left(x_{2}\right)<0$, so $H^{\prime \prime \prime}\left(x_{2}\right)$ is strictly decreasing. As

$$
H^{\prime \prime \prime}\left(x_{2}\right)<H^{\prime \prime \prime}(1 / 3)=54-54 \times 9 \times \frac{1}{8}=-\frac{27}{4}<0,
$$

$H^{\prime \prime}\left(x_{2}\right)$ is strictly decreasing. Since $H^{\prime \prime}\left(\frac{1}{3}\right)=\frac{3}{2}>0$ and $H^{\prime \prime}\left(\frac{5}{6}\right)=-\frac{18}{7}<0$, there is an $x_{2}^{0} \in$ $\left(\frac{1}{3}, \frac{5}{6}\right)$ such that $H^{\prime \prime}\left(x_{2}^{0}\right)=0, H^{\prime}\left(x_{2}\right)$ is strictly increasing for $x_{2} \in\left(\frac{1}{3}, x_{2}^{0}\right)$ and $H^{\prime}\left(x_{2}\right)$ is strictly decreasing for $x_{2} \in\left(x_{2}^{0}, \frac{5}{6}\right)$. As $H^{\prime}\left(\frac{1}{3}\right)=0$ and $H^{\prime}\left(\frac{5}{6}\right)=-\frac{1}{4}<0$, there is $a \in\left(x_{2}^{0}, \frac{5}{6}\right)$ such that $H^{\prime}(a)=0, H\left(x_{2}\right)$ is strictly increasing for $x_{2} \in\left(\frac{1}{3}, a\right)$ and $H\left(x_{2}\right)$ is strictly decreasing for $x_{2} \in$ $\left(a, \frac{5}{6}\right)$. Thus, for $x_{2} \in\left(\frac{1}{3}, \frac{5}{6}\right)$,

$$
H\left(x_{2}\right)>\min \{H(1 / 3), H(5 / 6)\}=\min \{0,5 / 216\}=0 .
$$

Hence, (26) holds and (24) follows from (26).

Example 2. Consider the three-dimensional Lotka-Volterra system

$$
\begin{aligned}
& \dot{x}_{1}=x_{1} f_{1}(x) \equiv x_{1}\left(1-x_{1}-\alpha x_{2}\right), \\
& \dot{x}_{2}=x_{2} f_{2}(x) \equiv x_{2}\left(1-x_{2}-\alpha x_{3}\right), \\
& \dot{x}_{3}=x_{3} f_{3}(x) \equiv x_{3}\left(1-x_{3}-\alpha x_{1}\right),
\end{aligned}
$$

where $\alpha>1$ is a parameter.

Proposition 4.4. System (31) with $\alpha>1$ has a unique carrying simplex $\Sigma$ and a unique interior equilibrium $p \in$ int $\Sigma$ with the following properties. 
(a) If $1<\alpha<2$, then $p$ is globally asymptotically stable in $\dot{C}$ and $\partial \Sigma$ is a heteroclinic limit cycle globally repelling on $\Sigma: \alpha(x)=\partial \Sigma$ for all $x \in \operatorname{int} \Sigma \backslash\{p\}$.

(b) If $\alpha=2$, then $\Sigma=\Delta^{2}=\left\{x \in C: x_{1}+x_{2}+x_{3}=1\right\}$, every trajectory in int $\Sigma \backslash\{p\}$ is a simple closed curve, $\partial \Sigma$ is a heteroclinic cycle, and $p$ is a centre on $\Sigma$.

(c) If $\alpha>2$, then $p$ is a saddle point with int $\Sigma \backslash\{p\}=W^{u}(p)$ the unstable manifold and $W^{s}(p)=\{k p: k>0\}$ the stable manifold. Further, $\partial \Sigma$ is a heteroclinic limit cycle globally attracting in $\dot{C}: \omega(x)=\partial \Sigma$ for all $x \in \dot{C} \backslash W^{s}(p)$.

Proof. Clearly, (31) meets the conditions of theorem 2.2 with $r=(1.1,1.1,1.1)$, so it has a unique carrying simplex $\Sigma$. We can check that $p=\left(\frac{1}{1+\alpha}, \frac{1}{1+\alpha}, \frac{1}{1+\alpha}\right)$ is the unique equilibrium in $\dot{C}$ and $e_{1}, e_{2}, e_{3}$ are the only equilibria on $\partial \Sigma$. Let

$$
V(x)=x_{1} x_{2} x_{3}, \quad W(x)=x_{1}+x_{2}+x_{3} .
$$

Then

$$
\begin{aligned}
\left.\dot{V}\right|_{(31)} & =V(x)[3-(1+\alpha) W(x)], \\
\left.\dot{W}\right|_{(31)} & =W(x)-W(x)^{2}+(2-\alpha)\left(x_{1} x_{2}+x_{2} x_{3}+x_{3} x_{1}\right) .
\end{aligned}
$$

Let

$$
\pi_{p}=\left\{x \in C: x_{1}+x_{2}+x_{3}=\frac{3}{1+\alpha}\right\} .
$$

Then, for $x \in \pi_{p}$,

$$
\left.\dot{W}\right|_{(31)}=(2-\alpha)\left[x_{1} x_{2}+x_{2} x_{3}+x_{3} x_{1}-\frac{3}{(1+\alpha)^{2}}\right] .
$$

We first show that

$$
\forall x \in \pi_{p} \backslash\{p\}, \quad x_{1} x_{2}+x_{2} x_{3}+x_{3} x_{1}<\frac{3}{(1+\alpha)^{2}} .
$$

Since $x_{1}=\frac{3}{1+\alpha}-x_{2}-x_{3}$ for $x \in \pi_{p}$, by letting

$$
G\left(x_{2}, x_{3}\right)=\left(\frac{3}{1+\alpha}-x_{2}-x_{3}\right)\left(x_{2}+x_{3}\right)+x_{2} x_{3},
$$

to show (37) we need only show that

$$
\forall x_{3} \in\left[0, \frac{3}{1+\alpha}\right], \forall x_{2} \in\left[0, \frac{3}{1+\alpha}-x_{3}\right], \quad G\left(x_{2}, x_{3}\right)<\frac{3}{(1+\alpha)^{2}}
$$

provided $\left(x_{2}, x_{3}\right) \neq\left(\frac{1}{1+\alpha}, \frac{1}{1+\alpha}\right)$. As $\frac{\partial G}{\partial x_{2}}=\frac{3}{1+\alpha}-2 x_{2}-x_{3}$, we have $\frac{\partial G}{\partial x_{2}}=0$ so $G\left(x_{2}, x_{3}\right)$ has maximum at $x_{2}=\frac{1}{2}\left(\frac{3}{1+\alpha}-x_{3}\right)$. Let $h\left(x_{3}\right)=G\left(\frac{1}{2}\left(\frac{3}{1+\alpha}-x_{3}\right), x_{3}\right)$. Then $h\left(x_{3}\right)$ is simplified as

$$
h\left(x_{3}\right)=-\frac{3}{4}\left[\left(x_{3}-\frac{1}{1+\alpha}\right)^{2}-\left(\frac{2}{1+\alpha}\right)^{2}\right]
$$

From this we see that, for $x_{3} \in\left[0, \frac{1}{1+\alpha}\right) \cup\left(\frac{1}{1+\alpha}, \frac{3}{1+\alpha}\right]$,

$$
h\left(x_{3}\right)<h(1 /(1+\alpha))=\frac{3}{(1+\alpha)^{2}} .
$$


As $x_{2}=\frac{1}{2}\left(\frac{3}{1+\alpha}-x_{3}\right)=\frac{1}{1+\alpha}$ when $x_{3}=\frac{1}{1+\alpha}$, we have shown that $G\left(x_{2}, x_{3}\right)<G\left(\frac{1}{1+\alpha}, \frac{1}{1+\alpha}\right)=$ $\frac{3}{(1+\alpha)^{2}}$, so that (38) holds, if $\left(x_{2}, x_{3}\right) \neq\left(\frac{1}{1+\alpha}, \frac{1}{1+\alpha}\right)$.

(a) When $1<\alpha<2$, from (36) and (37) we see that $\left.\dot{W}\right|_{(31)}<0$ for $x \in \pi_{p} \backslash\{p\}$. This shows that $\Sigma \backslash\{p\}$ is strictly below $\pi_{p}$. From (33) we have $\left.\dot{V}\right|_{(31)}>0$ for $x \in \operatorname{int} \Sigma \backslash\{p\}$. Thus, as $\gamma^{+}(x) \subset \operatorname{int} \Sigma \backslash\{p\}, V\left(\varphi_{t}(x)\right)$ is strictly increasing. By the same technique as that used in the proof of proposition 4.3, we have $\lim _{t \rightarrow+\infty} V\left(\varphi_{t}(x)\right)=V(p)$ and $\lim _{t \rightarrow+\infty} \varphi_{t}(x)=p$. By the definition of $\Sigma, p$ is globally attracting in $\dot{C}$. The Jacobian matrix

$$
J(p)=\left(\begin{array}{ccc}
-\frac{1}{1+\alpha} & -\frac{\alpha}{1+\alpha} & 0 \\
0 & -\frac{1}{1+\alpha} & -\frac{\alpha}{1+\alpha} \\
-\frac{\alpha}{1+\alpha} & 0 & -\frac{1}{1+\alpha}
\end{array}\right)
$$

has eigenvalues

$$
\lambda_{1}=-1, \quad \lambda_{2,3}=-\frac{2-\alpha}{2(1+\alpha)} \pm i \frac{\sqrt{3} \alpha}{2(1+\alpha)} .
$$

As $1<\alpha<2$ implies $\operatorname{Re} \lambda_{j}<0$ for all $j \in I_{3}, p$ is globally asymptotically stable in $\dot{C}$. Since

$$
\begin{aligned}
& f_{1}\left(e_{2}\right)=f_{2}\left(e_{3}\right)=f_{3}\left(e_{1}\right)=1-\alpha<0, \\
& f_{3}\left(e_{2}\right)=f_{1}\left(e_{3}\right)=f_{2}\left(e_{1}\right)=1>0,
\end{aligned}
$$

and $e_{1}, e_{2}, e_{3}$ are the only equilibria on $\partial \Sigma$, by theorem $4.2, \partial \Sigma$ is a heteroclinic limit cycle globally repelling on $\Sigma$.

(c) When $\alpha>2$, from (39) we see that $\lambda_{1}<0$ but $\operatorname{Re} \lambda_{2,3}>0$, so $p$ is a saddle point. Since $\{k p: k>0\}$ is a one-dimensional invariant manifold tangent at $p$ to an eigenvector, $(1,1,1)$, of $J(p)$ corresponding to the unique eigenvalue with negative real part, $\lambda_{1}=-1$, it is equal, by the uniqueness of the stable manifold, to $W^{s}(p)$.

We next show that $W^{u}(p)=$ int $\Sigma \backslash\{p\}$. From (36) and (37) we see that $\left.\dot{W}\right|_{(31)}>0$ for $x \in \pi_{p} \backslash\{p\}$ so that $\Sigma \backslash\{p\}$ is strictly above $\pi_{p}$. For each $x \in$ int $\Sigma \backslash\{p\}$, from (33) we have $\left.\dot{V}\right|_{(31)}<0$. As int $\Sigma \backslash\{p\}$ is invariant, $V\left(\varphi_{t}(x)\right)$ is a strictly decreasing function for $t \in \mathbb{R}$. As $V$ is bounded and positive on int $\Sigma$, both $\lim _{t \rightarrow+\infty} V\left(\varphi_{t}(x)\right)$ and $\lim _{t \rightarrow-\infty} V\left(\varphi_{t}(x)\right)$ exist. Hence, there are constants $a$ and $b$ satisfying $0 \leqslant a<V(x)<b$ such that $V(y)=a$ for all $y \in \omega(x)$ and $V(z)=b$ for all $z \in \alpha(x)$. If $\alpha(x) \neq\{p\}$, then, as $p$ is the unique interior equilibrium and $\alpha(x) \subset$ int $\Sigma$, there is a point $z \in \alpha(x) \backslash\{p\}$ that is not an equilibrium. As $\gamma(z) \subset \alpha(x)$, we should have $V\left(\varphi_{t}(z)\right)=b$ for all $t \in \mathbb{R}$, which contradicts the fact that $V\left(\varphi_{t}(z)\right)$ is strictly decreasing. This contradiction shows that $\alpha(x)=\{p\}$ and $b=V(p)$, hence, by the hyperbolicity of $p$, we must have $x \in W^{u}(p)$. On the other hand, for each $x \in W^{u}(p)$, its orbit $\gamma(x)$ is bounded, so, by lemma 3.1, $x \in A$. Suppose to the contrary that $x \notin \Sigma$. As $A=\Sigma \cup \mathcal{B}(0)$, we must have $x \in \mathcal{B}(0)$, so $\alpha(x)=\{0\}$, a contradiction. This shows that $W^{u}(p) \subset \Sigma$. It then follows from this and $W^{u}(p) \subset \dot{C} \backslash W^{s}(p)$ that $W^{u}(p) \subset$ int $\Sigma \backslash\{p\}$. Hence, $W^{u}(p)=$ int $\Sigma \backslash\{p\}$.

It then follows that $\omega(x) \subset \partial \Sigma$ for all $x \in W^{u}(p)$ so that $p$ repels int $\Sigma \backslash\{p\}$ to the boundary of $\Sigma$. By theorem $4.2, \partial \Sigma$ is a heteroclinic limit cycle globally attracting in $\dot{C}$. 
(b) When $\alpha=2$, from (32)-(36) we see that $\pi_{p}=\Delta^{2}$ and

$$
\forall x \in \Delta^{2},\left.\quad \dot{V}\right|_{(31)}=0,\left.\quad \dot{W}\right|_{(31)}=0 .
$$

Thus,

$$
\forall x \in \Delta^{2}, \forall t \in \mathbb{R}, \quad V\left(\varphi_{t}(x)\right)=V(x), \quad W\left(\varphi_{t}(x)\right)=1 .
$$

Equivalently,

$$
\forall x \in \Delta^{2}, \quad \gamma(x) \subset \pi_{p} \cap\{y \in C: V(y)=V(x)\} .
$$

If $x \in \Delta^{2} \backslash\{p\}$ with $x \gg 0$, then $V(x)>0$ and $\pi_{p} \cap\{y \in C: V(y)=V(x)\}$ is a simple closed curve. As $p$ is the only equilibrium in $\dot{C}$, there is no equilibrium on this curve. Hence, we must have $\gamma(x)=\pi_{p} \cap\{y \in C: V(y)=V(x)\}$. Since all closed trajectories and nontrivial equilibria are in $\Sigma$, we have $\Sigma=\Delta^{2}$ and all trajectories in int $\Sigma \backslash\{p\}$ are simple closed curves. That $\partial \Sigma$ is a heteroclinic cycle follows from the same reason as that in the last few lines of (a) above.

\section{Conclusion}

For competitive Kolmogorov system (1), Hirsch's theorem 1.1 has been widely accepted with a great impact in the research field of population dynamics and dynamical systems. Its main virtue is that the dynamics of system in the space $C \backslash\{0\}$ can be described by the dynamics on the carrying simplex $\Sigma$ as a co-dimension one global attractor. But users of the theorem have to pay the high price of requiring the system to be totally competitive. Fortunately, we have managed to reduce the cost to minimum and proved our theorem 2.2 for the existence and uniqueness of a carrying simplex: we only require the system to have strong internal competition for each individual species, a much weaker condition than total competition. This is a significant improvement to theorem 1.1 and, therefore, to the existing theory for competitive differential systems.

As an example of potential applications of theorem 2.2, we have updated a result on existence of heteroclinic limit cycles and their global attraction or repulsion, which was originally established using theorem 1.1, and provided concrete systems with globally attracting (repelling) heteroclinic limit cycles. Without theorem 2.2 it is not possible to deal with these systems by using theorem 1.1 as it is not applicable to these systems due to its strong unmet conditions.

Similar to the above example, it is expected that many available results based on theorem 1.1 can be updated by using theorem 2.2. Moreover, due to the much weaker conditions of theorem 2.2 , a broader class of systems to which theorem 1.1 is not applicable can now be explored by the application of theorem 2.2. The following two problems are just such instances.

(a) The smoothness problem of the carrying simplex $\Sigma$ as a surface: $\Sigma$ in the trivial example given in section 2 is the upper boundary of $[0, r]$ and the smoothness of $f$ has no impact on the smoothness of $\Sigma$. What is the essential condition for $\Sigma$ to be $C^{1}$ ?

(b) In [24, 42], three-dimensional systems with plane nullclines are classified into 33 equivalent classes when the $3 \times 3$ matrix $A$ has positive entries only. Are there any new classes or features induced by reducing some of the positive off-diagonal entries of $A$ to zero?

As the concept, as well as results, on carrying simplex for discrete dynamical systems is a consequence of inspiration of theorem 1.1 for continuous systems, it is natural to rethink whether the current available results on carrying simplex for discrete dynamical systems can 
be significantly improved based on the idea of theorem 2.2. The author is delighted to announce that this can be done and will be published in a separate paper.

\section{Acknowledgments}

The author is grateful to the referees for the comments and suggestions adopted in this version of the paper.

\section{ORCID iDs}

Zhanyuan Hou (D) https://orcid.org/0000-0001-5429-1120

\section{References}

[1] Baigent S 2012 Convexity-preserving flows of totally competitive planar Lotka-Volterra equations and the geometry of the carrying simplex Proc. Edinb. Math. Soc. 55 53-63

[2] Baigent S 2013 Geometry of carrying simplices of 3-species competitive Lotka-Volterra systems Nonlinearity 26 1001-29

[3] Baigent S 2016 Convexity of the carrying simplex for discrete-time planar competitive Kolmogorov systems J. Differ. Equ. Appl. 22 609-20

[4] Baigent S 2019 Convex geometry of the carrying simplex for the May-Leonard map Discret. Contin. Dyn. Syst. B 24 1697-723

[5] Baigent S and Hou Z 2012 Global stability of interior and boundary fixed points for Lotka-Volterra systems Differ. Equ. Dyn. Syst. 20 53-66

[6] Ruiz-Herrera A 2013 Exclusion and dominance in discrete population models via the carrying simplex J. Differ. Equ. Appl. 19 96-113

[7] Hirsch M W 1982 Systems of differential equations which are competitive or cooperative: I. Limit sets SIAM J. Math. Anal. 13 167-79

[8] Hirsch M W 1985 Systems of differential equations that are competitive or cooperative: II. Convergence almost everywhere SIAM J. Math. Anal. 16 423-39

[9] Hirsch M W 1988 Systems of differential equations which are competitive or cooperative: III. Competing species Nonlinearity $151-71$

[10] Hirsch M W 2008 On existence and uniqueness of the carrying simplex for competitive dynamical systems J. Biological Dynamics 2 169-79

[11] Hou Z 1999 Global attractor in autonomous competitive Lotka-Volterra systems Proc. Am. Math. Soc. $1273633-42$

[12] Hou Z 2009 Global attractor in competitive Lotka-Volterra systems Math. Nachr. 282 995-1008

[13] Hou Z 2009 Vanishing components in autonomous competitive Lotka-Volterra systems J. Math. Anal. Appl. 359 302-10

[14] Hou Z 2009 Geometric method for a global repellor in competitive Lotka-Volterra systems Nonlinear Anal. Theory Methods Appl. 71 3587-95

[15] Hou Z 2013 Permanence, global attraction and stability Lotka-Volterra and Related Systems, Recent Development in Population Dynamics ed S Ahmad and I Stamova (Berlin: de Gruyter \& Co) pp 1-62

[16] Hou Z 2014 Permanence criteria for Kolmogorov systems with delays Proc. R. Soc. Edinburgh A 144 511-31

[17] Hou Z 2019 Geometric method for global stability and repulsion in Kolmogorov systems Dynam. Syst. 34 456-83

[18] Hou Z and Baigent S 2011 Fixed point global attractors and repellors in competitive Lotka-Volterra systems Dynamical Systems 26 367-90

[19] Hou Z, Baigent S and Baigent S 2013 Heteroclinic limit cycles in competitive Kolmogorov systems Discret. Contin. Dyn. Syst. A 33 4071-93 
[20] Hou Z and Baigent S 2015 Global stability and repulsion in autonomous Kolmogorov systems Commun. Pure Appl. Anal. 14 1205-38

[21] Jiang J, Mierczyński J and Wang Y 2009 Smoothness of the carrying simplex for discrete-time competitive dynamical systems: a characterization of neat embedding J. Differ. Equ. 246 1623-72

[22] Jiang J and Niu L 2014 The dynamical behavior on the carrying simplex of a three-dimensional competitive system: II. Hyperbolic structure saturation Int. J. Biomath. 071450002

[23] Jiang J and Niu L 2014 The theorem of the carrying simplex for competitive system defined on the n-rectangle and its application to a three-dimensional system Int. J. Biomath. 071450063

[24] Jiang J and Niu L 2017 On the validity of Zeeman's classification for three dimensional competitive differential equations with linearly determined nullclines J. Differ. Equ. 263 7753-81

[25] Niu L and Jiang J 2016 On the equivalent classification of three-dimensional competitive AtkinsonAllen models relative to the boundary fixed points Discret. Contin. Dyn. Syst. 36 217-44

[26] Jiang J and Niu L 2017 On the equivalent classification of three-dimensional competitive Leslie/Gower models via the boundary dynamics on the carrying simplex J. Math. Biol. 74 $1223-61$

[27] Jiang J, Niu L and Wang Y 2016 On heteroclinic cycles of competitive maps via carrying simplices J. Math. Biol. 72 939-72

[28] Jiang J, Niu L and Zhu D 2014 On the complete classification of nullcline stable competitive threedimensional Gompertz models Nonlinear Anal.: Real World Appl. 20 21-35

[29] Liang X and Jiang J 2003 The dynamical behaviour of type-K competitive Kolmogorov systems and its application to three-dimensional type-K competitive Lotka Volterra systems Nonlinearity $16785-801$

[30] Mierczynski J 1994 The C1 property of carrying simplices for a class of competitive systems of ODEs J. Differ. Equ. 111 385-409

[31] Mierczyński J 1999 On smoothness of carrying simplices Proc. Am. Math. Soc. 127 543-51

[32] Mierczyński J 1999 On peaks in carrying simplices Colloq. Math. 81 285-92

[33] Mierczyński J 1999 Smoothness of carrying simplices for three-dimensional competitive systems: a counterexample Dyn. Contin. Discrete Impuls. Syst. A 6 147-54

[34] Mierczyński J and Schreiber S J 2002 Kolmogorov vector fields with robustly permanent subsystems J. Math. Anal. Appl. 267 329-37

[35] Tineo A 2001 On the convexity of the carrying simplex of planar Lotka-Volterra competitive systems Appl. Math. Comput. 123 1-16

[36] Caifeng T and Jifa J 1998 The coexistence of a community of species with limited competition $J$. Math. Anal. Appl. 217 233-45

[37] Wang Y and Jiang J 2001 The general properties of discrete-time competitive dynamical systems $J$. Differ. Equ. 176 470-93

[38] Wang Y and Jiang J 2002 Uniqueness and attractivity of the carrying simplex for discrete-time competitive dynamical systems J. Differ. Equ. 186 611-32

[39] Yu Y, Wang W and Lu Z 2007 Global stability of Gompertz model of three competing populations J. Math. Anal. Appl. $334333-48$

[40] Zeeman E C and Zeeman M L 1994 On the convexity of carrying simplices in competitive Lotka-Volterra systems Lecture Notes in Pure and Appl. Math. 152 353-64

[41] Zeeman E C and Zeeman M L 2003 From local to global behaviour in competitive Lotka-Volterra systems Trans. Am. Math. Soc. 355 713-34

[42] Zeeman M L 1993 Hopf bifurcations in competitive three-dimensional Lotka-Volterra systems Dyn. Stab. Syst. 8 189-217

[43] Zeeman M L 1995 Extinction in competitive Lotka-Volterra systems Proc. Am. Math. Soc. 123 $87-96$ 